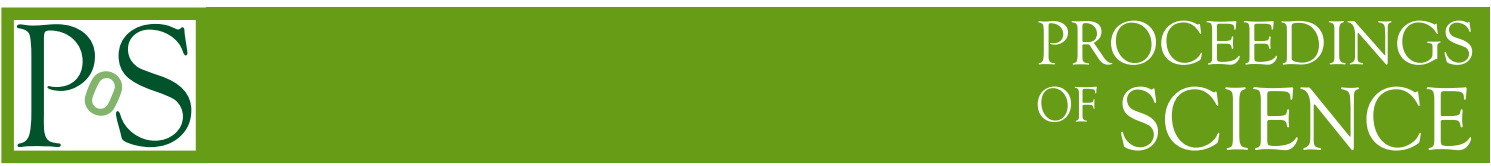

\title{
Advances in simulation and reconstruction for Hyper-Kamiokande
}

\section{N. W. Prouse* for the Hyper-Kamiokande Collaboration}

TRIUMF, Vancouver, British Columbia, Canada

E-mail: nprouse@triumf.ca

The next generation of neutrino experiments will require improvements to detector simulation and event reconstruction software matching the reduced statistical errors and increased and precision of new detectors. Here we present progress for the software of the Hyper-Kamiokande experiment being developed to enable reduction of systematic errors to below the $1 \%$ level. The current status and future prospects of this software is be presented, including advances in detector simulation and reconstruction using traditional techniques as well as new developments using modern machinelearning based approaches.

40th International Conference on High Energy physics - ICHEP2020

July 28 - August 6, 2020

Prague, Czech Republic (virtual meeting)

${ }^{*}$ Speaker 


\section{Introduction}

Hyper-Kamiokande [1] (HK) is the next generation water-Cherenkov neutrino experiment, building upon the success of Kamiokande, Super-Kamiokande (SK) and T2K. The physics program includes neutrino oscillation and neutrino astrophysics by observing accelerator, atmospheric, solar, and supernova neutrinos, as well as extended proton decay searches using the large amount of water in the tank. The experiment is planned to start operation in 2027 and will involve two new water Cherenkov detectors.

Construction has started recently on the far detector, consisting of a $258 \mathrm{kt}$ total (188 kt fiducial) volume of ultra-pure water surrounded by approx. $40 \%$ coverage of $50 \mathrm{~cm}$ PMTs. With about $8 \mathrm{x}$ larger fiducial volume than SK it will benefit from significantly increased statistics, but also new photosensor technology will provide improved photo-efficiency and timing resolution.

An intermediate water Cherenkov detector (IWCD), based on the nuPRISM proposal [2], is also planned to be constructed approx. $1 \mathrm{~km}$ from the source of the J-PARC neutrino beam, to measure the flux and cross-section of beam neutrinos using the same target and detector technologies as the far detector. The IWCD will consist of a $\sim 1 \mathrm{kt}$ volume of water with approx. 500 multi-PMT (mPMT) modules, each containing $198 \mathrm{~cm}$ PMTs, that provide improved position, direction and timing information over the $50 \mathrm{~cm}$ PMTs, allowing the smaller scale IWCD to achieve equivalent precision to the far detector. The IWCD detector volume will be able to move vertically within a $50 \mathrm{~m}$ tall pit, allowing measurements at different off-axis angles providing different neutrino energy spectra.

In order to exploit the new detector technologies and capabilities, improved simulation and reconstruction software is required, with development focusing on flexibility to simulate and reconstruct events with different detector geometries and configurations, to aid detector design optimisation studies and provide a consistent framework across different detectors.

\section{Simulation software}

The water Cherenkov detector simulation software of HK is called WCSim [3]. This opensource package is based on Geant4 [4] and provides a fast, flexible and consistent simulation framework where the detector geometry can be configured to allow its use for both the far detector and IWCD as well as other water Cherenkov detectors. The products of neutrino interactions, or of background events, are tracked through the detector, simulating all interactions within the detector volume, until any optical photons hit the PMT glass. The response of the PMTs to thse photons are then simulated along with the electronics, triggering and data acquisition. Both the true and digitised PMT hit times and charges are recorded, along with additional true particle tracking information, to be used in reconstruction software tuning as well as physics analysis. The triggering and DAQ simulations can also be simulated separately using the ToolDAQ [5] package, which is able to use the same code for simulation as is used in the actual DAQ electronics. 


\section{Reconstruction software}

\subsection{Low-energy reconstruction}

The reconstruction of low-energy events, where few PMTs are hit and each hit generally corresponds to only one photoelectron, is based on the hit timing information. Single Cherenkov rings are reconstructed assuming a point source of light, coming from the vertex position $(\vec{v})$ that maximises the following goodness function $g(\vec{v})$,

$$
g(\vec{v})=\sum_{i=1}^{N} w_{i} \exp \left(-0.5\left(\frac{t_{i}-\left|\vec{x}_{i}-\vec{v}\right| / c_{w a t}}{\sigma}\right)^{2}\right),
$$

where $t_{i}$ and $\vec{x}_{i}$ are the times and positions respectively of the $N$ hit PMTs, $w_{i}$ are Gaussian hit weights, $c_{\text {wat }}$ is the speed of light in water and $\sigma$ is the PMT timing resolution. The direction is reconstructed using a circular KS test of the pattern of hits around the Cherenkov cone, and the reconstructed energy scales with the number of hits observed around the expected timing at each PMT. The software used for SK, known as BONSAI, has been adapted and tuned for the HK detectors, while a new more modern and flexible framework, known as LEAF, is under development with a new optimisation algorithm but otherwise currently using the same reconstruction algorithms as BONSAI. Figure 1 shows the reconstructed position resolutions for different configurations of the far detector, each configuration having been simulated with WCSim and reconstructed using BONSAI.
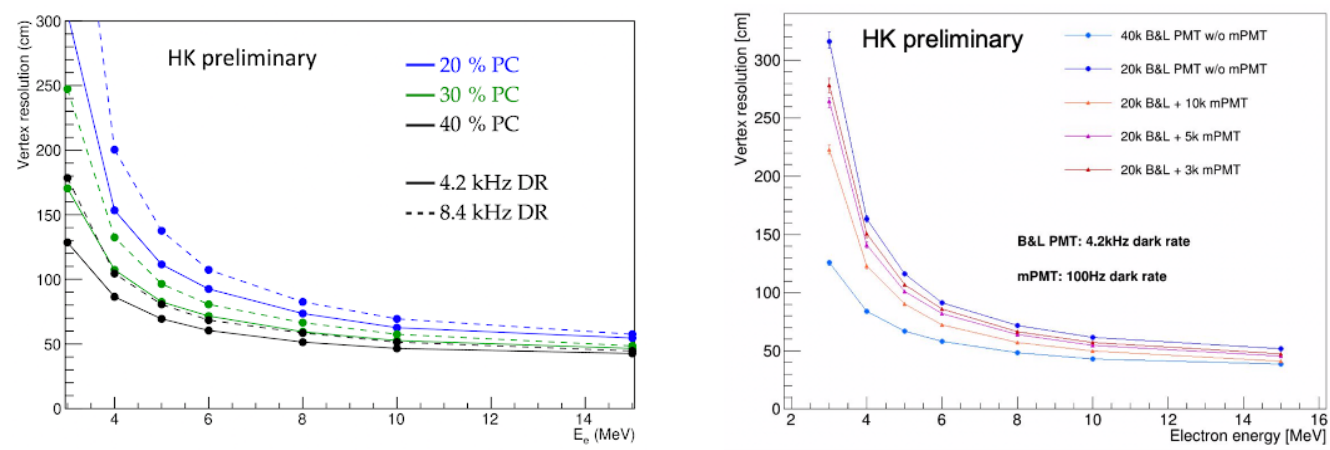

Figure 1: Low energy reconstructed position resolutions for various configurations of the HK far detector. Left: resolutions for 20\%, 30\% and 40\% photocoverage (PC) for PMTs with $4.2 \mathrm{kHz}$ or $8.4 \mathrm{kHz}$ dark rate (DR). Right: resolutions for various combinations of $50 \mathrm{~cm}$ box and line (B\&L) PMTs with mPMTs.

\subsection{High-energy reconstruction}

The high-energy event reconstruction software for HK is called fiTQun and was originally developed for SK, based on the algorithm used for MiniBooNE [6]. The event hypothesis $x$ comprises of the unknown position, direction and energy for a given particle type. These unknowns are reconstructed using the full information of time and charge from both unhit and hit PMTs, by finding the hypothesis $x$ that maximises the likelihood $L(x)$,

$$
L(x)=\prod_{j=1}^{N_{\text {unhit }}} P_{j}(\text { unhit } \mid x) \prod_{i=1}^{N_{\text {hit }}} P_{i}(\text { hit } \mid x) f_{q}\left(q_{i} \mid x\right) f_{t}\left(t_{i} \mid x\right),
$$


where $P_{j}($ unhit $\mid x)$ are the probabilities of the $N_{u n h i t}$ PMTs being unhit, $P_{i}($ hit $\mid x)$ are the probabilities of the $N_{\text {hit }}$ PMTs being hit, $f_{q}\left(q_{i} \mid x\right)$ are the probability densities of the hit PMTs' charges $q_{i}$ and $f_{t}\left(t_{i} \mid x\right)$ are the probability densities of the hit PMTs' times $t_{i}$, all assuming a given hypothesis $x$. Each of the probabilities in Eq. 2 is determined by combining the expected direct light as well as scattered and reflected light reaching each PMT. These are tuned to each detector geometry using simulated events for each particle type being considered, and the reconstructed particle type is determined using the ratio of the maximum likelihoods obtained when assuming each particle type. Figure 2 shows the reconstructed position resolutions for different configurations of the IWCD, each configuration having been simulated with WCSim and reconstructed using fiTQun.
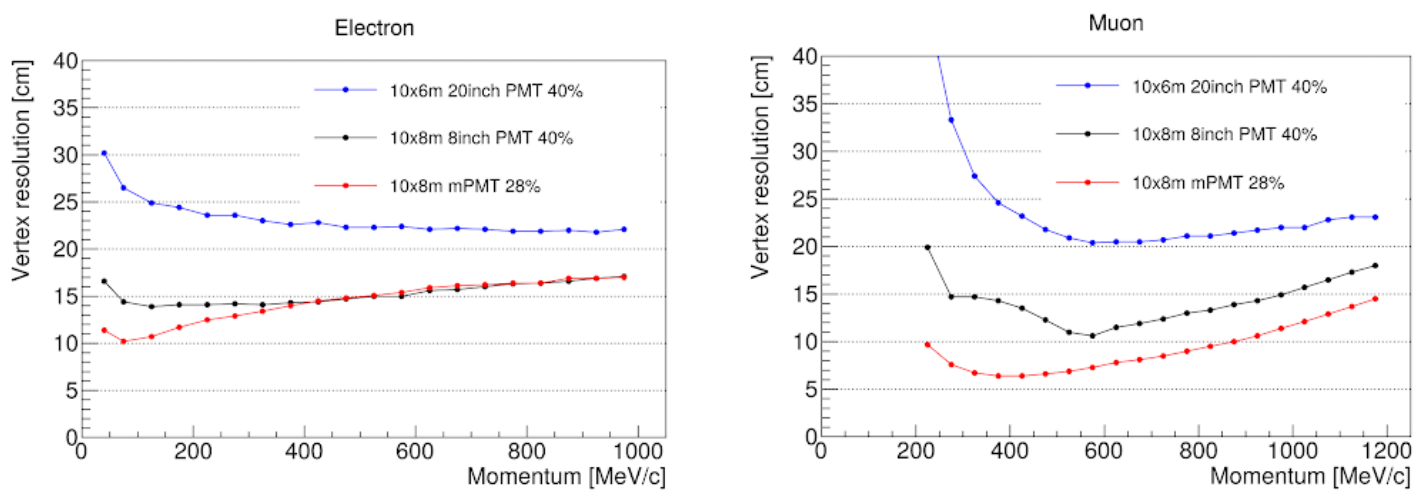

Figure 2: Resolutions for high-energy position reconstruction for electrons (left) and muons (right) for various configurations of the IWCD.

Neutral pions form a significant background when the $\pi^{0}$ decays to two largely co-linear gammas producing electromagnetic showers that appear similar to the signal from electrons produced by $v_{e}$ events. To distinguish neutral pions from electrons, a 2D cut is applied using the likelihood ratio together with the reconstructed $\pi^{0}$ mass. Figure 3 shows the separation of $\pi^{0}$ background and $v_{e}$ signal using these outputs from fiTQun.

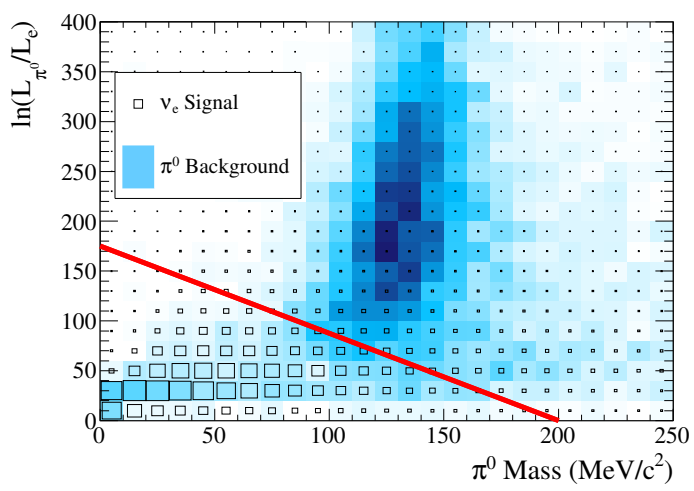

Figure 3: $\pi^{0}$ rejection cut (red line) with events above the line rejected as background against the $v_{e}$ signal 


\subsection{Machine Learning}

Traditional reconstruction software like fiTQun is beginning to reach the limit of the achievable reconstruction precision; to improve further, more complex likelihood functions would be required after relaxing some of the assumptions made in their current construction. But this would result in increased computational complexity, with reconstruction already the most intensive part of the full software chain. For larger detectors with a larger number of PMTs, the computation time is already increased and so alternative methods are being explored.

Machine learning (ML) has been revolutionary in the field of computer vision and is now also becoming common throughout HEP applications. ML has the potential for reconstruction to use all information without making physics assumptions beyond those of the simulation software used to train the models. Additionally, once trained these models use far less computational resources to reconstruct events. The WatChMaL organisation was formed to facilitate development of machine learning reconstruction for water Cherenkov detectors, including HK's far detector and IWCD. For the IWCD, initial studies have begin to explore particle type classification using various neural network architectures.

Inspired by the revolutionary success of convolutional neural networks (CNNs) in image processing [7], a CNN based on ResNet [8] has been developed to distinguish muon, electron and gamma events in the IWCD. The input to the network is a 40x40 pixel image containing both the barrel and end-caps mPMTs of the detector; each pixel corresponds to a single mPMT with 38 channels for the time and charge of each of the 19 PMTs. The network architecture is equivalent to the ResNet-18 architecture with an additional $1 \times 1$ pixel (single mPMT) convolution layer over the initial 38 channels, corresponding to a convolution of the times and charges of the 19 PMTs in each mPMT. The network was trained on 9 million simulated single-ring events, 3 million of each particle type, with uniform random energies from 0 to $1 \mathrm{GeV}$ above Cherenkov threshold, uniform random position inside the detector volume and isotropic random direction. Figure 4 demonstrates significantly improved discrimination of muon rings and electron rings using the ResNet CNN compared to fiTQun reconstruction, as well as some statistical separation of electron and gamma rings that fiTQun has not yet been able to successfully discriminate.
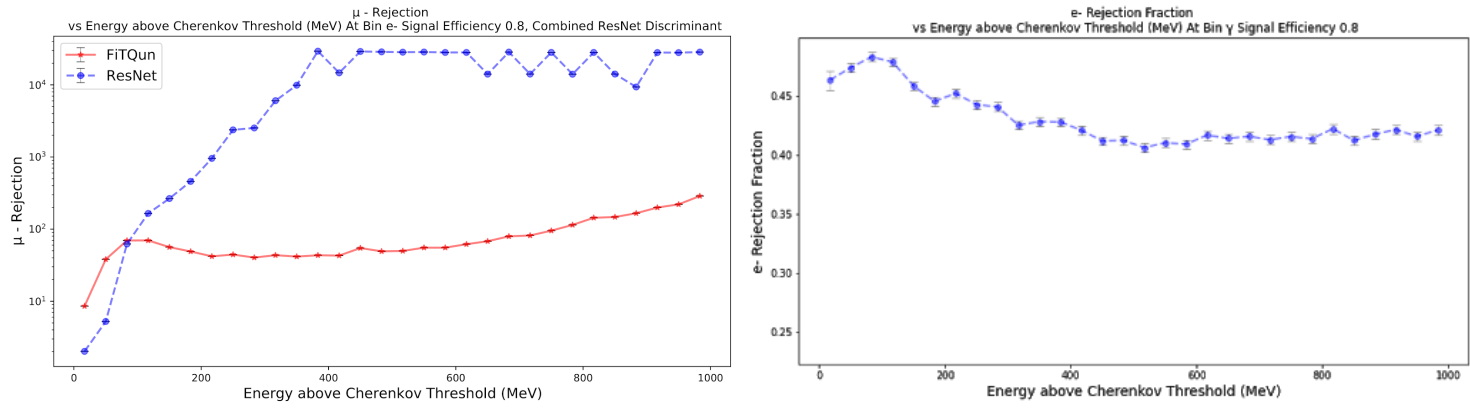

Figure 4: Left: Muon background rejection performance of ResNet-based CNN and fiTQun for fixed electron signal efficiency of $80 \%$. Right: Electron rejection performance of ResNet-based CNN with fixed gamma efficiency of $80 \%$.

These results using CNNs are encouraging, however the unwrapping of the cylindrical geometry onto a flat square image does not preserve the true geometrical topology of the detector, potentially 
limiting the ability of the network to generalise and provide optimal reconstruction performance. To address this, several methods are under investigation that correctly apply to the cylindrical geometry. The PointNet [9] architecture, which takes points (PMTs) in 3D space, together with features (time and charge) at each point has been adapted and is showing comparable performance to the ResNet CNN. Graph convolutional networks [10] are also being investigated, along with a newly developed method using a topology-preserving map of the cylindrical surface onto a rectangular grid image for input into a CNN architecture. The latter has been applied to reconstruction of energy, position and direction with initial results giving similar reconstruction performance to fiTQun. Additional development is underway towards the use of generative networks [11] for a hybrid reconstruction method using ML generated likelihoods with fiTQun-like reconstruction algorithms. Generative adversarial networks [12] are also being investigated for improving detector simulations and treatment of systematics.

\section{References}

[1] HyPer-KamioKANDE collaboration, Hyper-Kamiokande Design Report, 1805.04163.

[2] NUPRISM collaboration, Letter of Intent to Construct a nuPRISM Detector in the J-PARC Neutrino Beamline, 1412. 3086.

[3] WCSim, https://github.com/WCSim/WCSim. GitHub repository.

[4] GEANT4 collaboration, GEANT4-a simulation toolkit, Nucl. Instrum. Meth. A 506 (2003) 250.

[5] ToolDAQ, https://github.com/ToolDAQ/ToolDAQFramework. GitHub repository.

[6] R. Patterson, E. Laird, Y. Liu, P. Meyers, I. Stancu and H. Tanaka, The Extended-track reconstruction for MiniBooNE, Nucl. Instrum. Meth. A 608 (2009) 206 [0902 . 2222].

[7] Y. LeCun, Y. Bengio and G. Hinton, Deep learning, Nature 521 (2015) 436.

[8] K. He, X. Zhang, S. Ren and J. Sun, Deep residual learning for image recognition, 1512.03385

[9] R.Q. Charles, H. Su, M. Kaichun and L.J. Guibas, Pointnet: Deep learning on point sets for $3 d$ classification and segmentation, 1612.00593.

[10] T.N. Kipf and M. Welling, Semi-supervised classification with graph convolutional networks, 1609.02907.

[11] A. Dosovitskiy, J.T. Springenberg, M. Tatarchenko and T. Brox, Learning to generate chairs, tables and cars with convolutional networks, IEEE Transactions on Pattern Analysis and Machine Intelligence 39 (2017) 692 [1411.5928].

[12] I. Goodfellow, J. Pouget-Abadie, M. Mirza, B. Xu, D. Warde-Farley, S. Ozair et al., Generative adversarial nets, 1406.2661. 